Journal of Applied Fluid Mechanics, Vol. 15, No. 2, pp. 325-336, 2022.

Available online at www.jafmonline.net, ISSN 1735-3572, EISSN 1735-3645.

https://doi.org/10.47176/jafm.15.02.32653

\title{
Investigation of Isothermal Flow inside a New Combustor with Two-Stage Axial Swirler
}

\author{
P. Wang ${ }^{1 \dagger}$, X. Wei ${ }^{1}$, P. Shrotriya ${ }^{2}$, W. $\operatorname{Li}^{1}$ and A. Ferrante ${ }^{1,3}$ \\ ${ }^{1}$ Institute for Energy Research of Jiangsu University, Zhenjiang, 212013, China \\ ${ }^{2}$ School of Energy and Power Engineering, Jiangsu University, Zhenjiang 212013, China \\ ${ }^{3}$ Combustion Environment Research Centre, 70023, Italy \\ †Corresponding Author Email: pingwang@ujs.edu.cn
}

(Received February 2, 2021; accepted August 17, 2021)

\begin{abstract}
Experiments and numerical simulations are performed to study the cold flow field characteristics in a two-stage axial swirl combustion chamber. Large eddy simulation with dynamic turbulent kinetic energy sub-grid scale model is used to calculate the flow field, and particle image velocimetry is used to measure the turbulent flow field. The calculated results are found to be in a good agreement with the experiment results. Due to the shear layers and the density difference between $\mathrm{CO}_{2}$ and air flows, the central recirculation zone appears. With the increase of the ratio of flow velocity between inner and outer tubes, the central recirculation zone shrinks gradually, while the length and range of the angular recirculation zone increase continuously. The vortex structure develops in the axial and radial directions, and the vortex breakdown mostly occurs in the upstream regions. However, outside the central recirculation zone, only one shear layer is observed, and its vortex structure is almost extended up to the exit of the combustion chamber. In addition, precession vortex core is not seen in all the conditions.
\end{abstract}

Keywords: Double swirl; Particle Image Velocimetry; Large eddy simulation; Coherent structure; Vortex breakdown.

\section{NOMENCLATURE}

$D$

outer diameter of the swirler

inner diameter of the swirler

inner tubes

outer tubes

volume flow rate

\section{$R$}

$S$

$R e$

$\theta$ velocity ratio of the inner tube to the outer tube swirl number Reynolds number geometric blade Angle

\section{Introduction}

Swirlers are commonly used to improve and control the mixing process between fuel and air streams and to enhance flame stability, heat release rate, and consequently, to achieve low $\mathrm{NO}_{\mathrm{x}}$ emission (Eldrainy et al. 2011, Beér et al. 1972). Single-stage swirler has been widely studied over the last decade. Some studies on the single-stage swirler have shown the influence of its structure on flow field (Raj and Ganesan 2008, Cai et al. 2005). Measurements of a single-stage swirler flow field in rectangular expansive combustion chamber with particle image velocimetry (PIV) were performed to analyze the averaged velocity, root mean square fluctuation of velocity and Reynolds shear stresses (Reddy et al. 2006, Kim et al. 2009). They observed central and angular recirculation zones in a chamber, and the connection between installation angle of swirl vane and combustion flow field characteristics. In addition, some studies used large eddy simulation (LES) numerical method to study vortex breakdown and the connection between the increase of the number of swirling currents and the movement of the recirculation zones (Wu et al. 2016, Lu et al. 2005, Huang and Yang 2005).

However, the use of two-stage swirler have been 
P. Wang et al. / JAFM, Vol. 15, No. 2, pp. 325-336, 2022

significantly increased in the cold flow field studies since their ability to meet the characteristics of higher temperature and heavy loads compared with singlestage swirler. Measurement of the flow field of a double axial swirler with PIV was performed by Archer and Gupta (2004). They stated that under noncombustion conditions, both axial and radial velocities were affected by the rotational flow distribution within the burner, and in turn, radial distribution of the rotational flow had a significant influence on the flow field. Moreover, the inertia of fuel tended to establish a more symmetrical flow field for the vortex structure. Manikandan et al. (2020) has shown that the flame became shorter and wider with increase in swirl number.

Multi-gas analyzer for Fourier transform infrared (FTIR) spectroscopy and dual-phase Doppler anemometry (PDA) were used to measure the state of flow field (El-Kady et al. 2005, 2006, Hadef and Lenze 2008), yielding significant information about the formation of nitric oxide and carbon monoxide during swirling combustion, and also the increase in turbulence intensity produced by the structure of counter-swirl.

The results of iso-surface with zero velocity show that the flow structure is asymmetrically distributed in the entire combustor, and the flow trend on one side of the swirler is relatively high (Vashahi et al. 2017a, 2017b). LES analysis on a new type of variable vane swirler for gas turbine combustion chamber was performed by Eldrainy et al. (Eldrainy et al. 2011). LES methods are very effective for studying various turbulence characteristics of swirl stabilized combustor. They can be effectively used for treatment and studying dissipation of vortex breakdown and swirl structure, as well as angular recirculating zone and central recirculating zone, important characteristics of swirl (Kim et al. 2011, Tiwari et al. 2020). Canepa et al. (2006) has shown that the complex flow field structure comes from vortex breakdown. The vortex breakdown causes the formation of spiral PVC, which develops in downstream locations along the axial and radial directions. Flow field characteristics of different types of swirler have been studied widely, however, the research methods used in different studies were relatively simple, either measured by experimental means or calculated by numerical simulation only. RANS analysis on swirling field for studying the vortex position was performed by Houben et al. (2016).

The present work studies a self-designed two-stage axial swirler which contains an inner swirler and an outer swirler, which is mounted at the bottom of the burner. In order to systematically study the flow field characteristics in the chamber, both experiments and LES methods are employed and their results are analyzed and compared to each other.

\section{Experimental setup}

The experimental setup used in this work is shown in
Fig.1 (a). It consists of inner and outer tubes, whose diameters are $11 \mathrm{~mm}$ and $21 \mathrm{~mm}$, respectively. The wall thicknesses of both tubes are $1 \mathrm{~mm}$. Square combustion chamber with height of $142 \mathrm{~mm}$ and width of $120 \mathrm{~mm}$ is fixed by stainless-steel supports, and it is sealed by quartz glass for measuring the entire flow field with optical diagnostic system. The exhaust gas outlet is designed to greatly eliminate the upstream adverse pressure (Wu et al. 2016). Corotating double swirler is fixed between tubes and chamber, as shown in Fig.1 (b). Swirler has 8 internal and 8 external blades. Both types of blades rotate in the same directions, and they are twisted at 120 degrees along the central axis.

The thickness of blades is $0.6 \mathrm{~mm}$ and swirl number is 0.53 , which is calculated according to equation by (1) (Beer and Chigier 1972)

$$
S=\frac{2\left(1-\left(\frac{d}{D}\right)^{3}\right)}{3\left(1-\left(\frac{d}{D}\right)^{2}\right)} \tan \theta
$$

where $\mathrm{S}, \mathrm{d}, \mathrm{D}$ and $\theta$ represent swirl number, the inner diameter of the swirler, outer diameter of the swirler and the geometric blade angle, respectively.

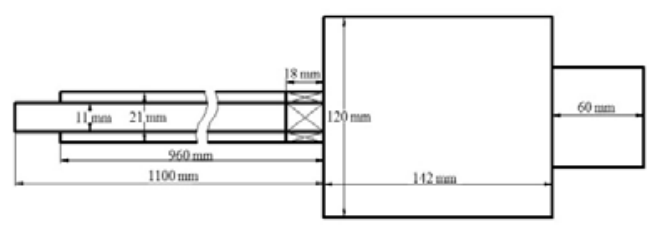

(a)

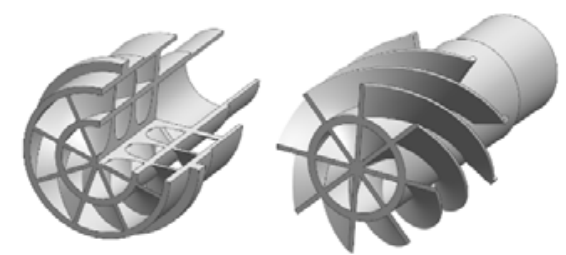

(b)

Fig. 1 (a) Schematic of the double swirl burner, (b) Isometric view of the two-stage axial swirler.

Two-dimensional PIV, a dominant method for velocimetry in experimental fluid mechanics (Westerweel et al. 2013, Arthur 2018, Adawy et al. 2018), is applied to measure the velocity field of the swirling jets. The experimental configuration is shown in Fig. 2. The continuous light source used is a Nd:YAG laser with $532 \mathrm{~nm}$ output wavelength, 200 $\mathrm{mJ}$ monopulse, adjustable repetition frequency 1-15 $\mathrm{Hz}$ and energy instability less than 3 percent. The 
lamination system converts the spot into a sheet, which passes through the test section for further analysis. $\mathrm{TiO}_{2}$ particles with diameters of $2 \mu \mathrm{m}$ are dispersed in the both tubes and chamber as seeds. The visualized images are recorded as consecutive images by a CCD high-speed video camera (Fastcam SA5, Photron) at $15 \mathrm{fps}$ with a frame size of $2056 \times 2056$ pixels. The spatial resolution of image is $7.4 \times 7.4 \mu \mathrm{m}$ per pixel. $\mathrm{TiO}_{2}$ particles are caught in 12 bit grayscale. Aperture of $\mathrm{f} 2$ and double pulse laser of $200 \mathrm{mj}$ are set to enhance the signal of flow field itself, improving the scattering of tracer particles and the SNR (Signal to noise ratio) (Stopper et al. 2009).

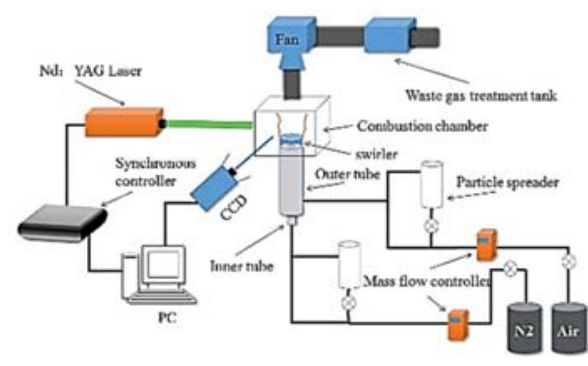

Fig. 2. Schematic of the experimental configuration.

\section{Numerical setup}

LES is used in this work for turbulent flow field prediction (Yang 2012). In this method, after filtering the Navier Stokes equations, the large-scale eddies are computed while the small-scale eddies are modeled. In this way, one can save some computing resources used for simulating the generation and evolution of vortex structure in the flow field. The dynamic turbulent kinetic energy sub-grid scale (SGS) turbulence model is used to estimate SGS stresses.

The simulation is carried out in the open-source CFD toolkit OpenFOAM (Open-source Field Operation And Manipulation). More precisely, PIMPLE algorithm was adopted, and its dictionary file contains algorithm control information. The "interDyMFOAM" solver was adopted, which is usually used for combustion flow fields, but can be applied in isothermal flow fields as well.
In order to improve the calculation efficiency, the length of the inlet pipe is set as $28 \mathrm{~mm}$, and the turbulent velocity inlet boundary condition is adopted at the inlet boundary, i.e., a certain turbulent kinetic energy dissipation rate and velocity fluctuations are set at the inlet boundary. So that the flow can be fully developed after entering the pipe, and it can reach the turbulent state at the outlet of the pipe. Additionally, the inlet condition for the velocity is set as "TurbulentInlet", which is a boundary condition available in the OpenFOAM. This boundary condition generates a fluctuation in the inlet velocity by adding a random disturbance in the mean velocity. In all cases the $3 \mathrm{D}$ computational meshes are generated using ICEM-CFD software. To obtain high quality meshes, structured hexahedral grids are generated. A grid resolution study is carried out to determine a suitable mesh from 3 meshes with different number of cells. Finally, a grid with 1.7 million grid cells have been chosen to conduct the hereafter simulations.

\section{Result}

In the present study, four different cases are considered, based on the flow of air and $\mathrm{CO}_{2}$ in the outer and inner tubes. In the case $1, \mathrm{CO}_{2}$ is injected into inner tube with velocity of $U_{I}=10.15 \mathrm{~m} / \mathrm{s}$, and air is injected into outer tube with velocity of $U_{O}=$ $16.04 \mathrm{~m} / \mathrm{s}$. In the case 2 , air and $\mathrm{CO}_{2}$ are flow into inner and outer tubes with velocities of $U_{I}=19.12$ $\mathrm{m} / \mathrm{s}$ and $U_{O}=8.52 \mathrm{~m} / \mathrm{s}$, respectively.

In addition, the velocity ratios of the inner tube to the outer tube are largely effected by injecting gas species under the condition of constant volume flow through the inner and outer tubes. In the case 3, air is injected into inner tube with velocity of $U_{I}=10 \mathrm{~m} / \mathrm{s}$, $\mathrm{CO}_{2}$ is injected into outer tube with velocity of $U_{O}=10 \mathrm{~m} / \mathrm{s}$. For the case $4, \mathrm{CO}_{2}$ is injected into inner tube with velocity of $U_{I}=22.48 \mathrm{~m} / \mathrm{s}$, air is injected into outer tube with velocity of $U_{O}=4.45 \mathrm{~m} / \mathrm{s}$. Major flow parameters of all the cases are shown in Table 1, where the symbols $R e$ and $Q$ denote Reynolds number and volume flow rate, respectively, and the subscripts ' $\mathrm{I}$ ' and ' $\mathrm{O}$ ' represent the inner and outer tubes. " $R$ " denotes the velocity ratio of the inner tube to the outer tube. Axial velocity, radial velocity, rms velocity, shear stress and coherent structure obtained from experiments and LES will be discussed in the following section.

Table 1 Inlet conditions for the four cases

\begin{tabular}{|c|c|c|c|c|c|c|c|c|c|}
\hline case & Inner tube & $\mathrm{U}_{\mathrm{I}}(\mathrm{m} / \mathrm{s})$ & $\mathrm{Q}_{\mathrm{I}}(\mathrm{NL} / \mathrm{min})$ & $R e_{\mathrm{I}}$ & Outer tube & $U_{O}(\mathrm{~m} / \mathrm{s})$ & $Q_{0}(\mathrm{NL} / \mathrm{min})$ & $R e_{\mathrm{O}}$ & $R$ \\
\hline 1 & $\mathrm{CO}_{2}$ & 10.15 & 53 & 13300 & Air & 16.04 & 188.4 & 8120 & 0.63 \\
\hline 2 & Air & 19.12 & 99.83 & 13300 & $\mathrm{CO}_{2}$ & 8.52 & 100 & 8120 & 2.24 \\
\hline 3 & Air & 10 & 52.22 & 6374 & $\mathrm{CO}_{2}$ & 10 & 117.37 & 8732 & 1 \\
\hline 4 & $\mathrm{CO}_{2}$ & 22.48 & 117.37 & 29448 & 8.52 & 4.45 & 52.22 & 2250 & 5.05 \\
\hline
\end{tabular}




\subsection{Influence of $\mathrm{Re}$}

The experimental flow field information of the case 1 related to mean axial and radial velocities are shown in Fig. 3(a), where the black and white solid lines in the axial velocity field represent the isosurface of zero velocity obtained from experiments and LES, respectively. The iso-surface of zero velocity denotes the boundary of the recirculation zone. It is observed that a "funnel" shaped region is formed in the whole combustion chamber, which is often referred to as the central recirculation zone. As it develops along the downstream, it reaches the middle of the combustor $(x=75 \mathrm{~mm})$ and its widest point is up to $100 \mathrm{~mm}$ away from the center of the combustor. On both side of the bottom of the burner, small negative velocity zones also present, which are angular recirculation zones. As the airflow reaches the wall, some part of the airflow is impacted by the wall and develops along the downstream direction, while the other part develops near the bottom of the combustion chamber.

In the case 2, the Reynolds number is same as the case 1 , but the inflow gases have been interchanged in the inner and outer tubes. Due to the difference of gas density and inflow velocity, the case 2 presents completely different velocity flow field characteristics, as shown in Fig. 3(b). After air comes out of the swirler, a rotating jet is formed in the combustion chamber. At the position close to the swirler, similar to the circular tube jet, the axial velocity is also relatively large. It can be seen from the iso-surface that in the upper and middle part of the combustion chamber, the central reflux region is discontinuous, and it shapes like "fragment". Compared with the central reflux region shown in the Fig. 3(a), the presence of central reflux in the case 2 is almost negligible. Such difference mainly comes from the large air velocity in the outer pipe for the case 1 , which results in large radial velocity at the exit of swirler. It produces strong adverse pressure gradients, which cause a strong entrainment of the flow to the central region, and more gas flow into the combustion chamber along the downstream directions. In turn, a wider recirculation zone appears. Furthermore, an angular recirculation zone in the case 2 is longer and wider compared to the case 1 .

In order to qualitatively analyze the flow field characteristics of the two cases, and to check the consistency between LES and experimental results, the values of the LES and experimental results on the central section of the swirler with axial distances of $10 \mathrm{~mm}, 20 \mathrm{~mm}, 40 \mathrm{~mm}, 80 \mathrm{~mm}$ and $120 \mathrm{~mm}$ from the exit of the swirler are compared in the Fig. 4, 5 and 6.

Figure 4 shows the calculated and measured averaged axial velocity along the radial direction for the two cases. It is observed that the shear effect in the case 1 gradually weakens along the downstream locations. The airflow in the inner and outer tubes mixes and forms a central recirculation zone. On the cross section of $x=120 \mathrm{~mm}$, axial velocity is close to zero, which means that this position is located in the end of center recirculation zone, leading to a reduction of the width of the recirculation zone.A good agreement is observed between the LES and experimental radial profiles of axial and velocities for both the cases. However, a little discrepancy observed between the experimental data and the LES calculation results is not negligible. Furthermore, LES results show some deviation from the experimental results at $x=10 \mathrm{~mm}$. This is possibly due to the not fine enough grid resolution over there. A strong shear layer is generated between the inner and outer swirling flows, which requires a very fine grid to resolve it.

Figure 5 shows the radial profiles of rms axial velocities for the case 1 and case 2 at different axial locations. The values of the rms velocity reflect the statistical results of the turbulent fluctuations.

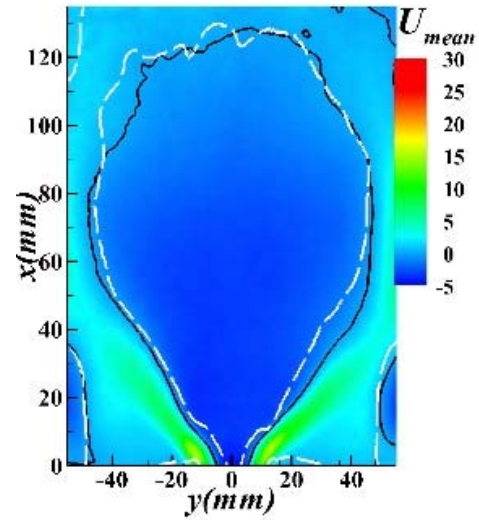

(a)

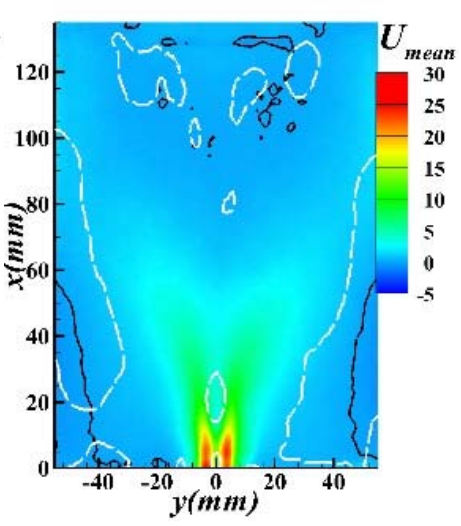

(b)

Fig. 3. Contour of averaged axial velocity. (a) for the case 1. (b) for the case 2. 


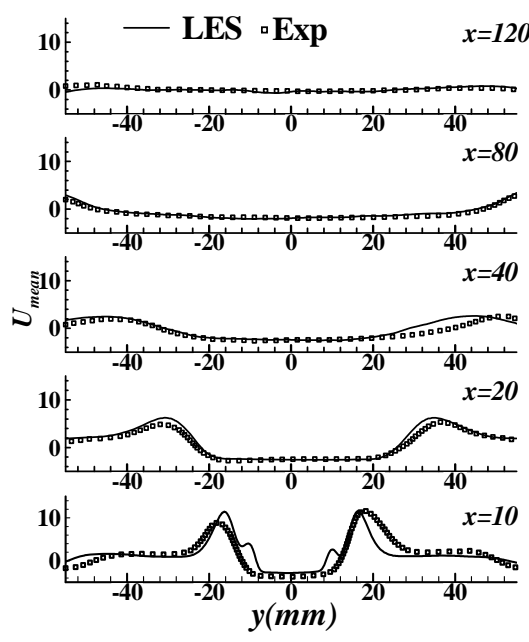

(a)

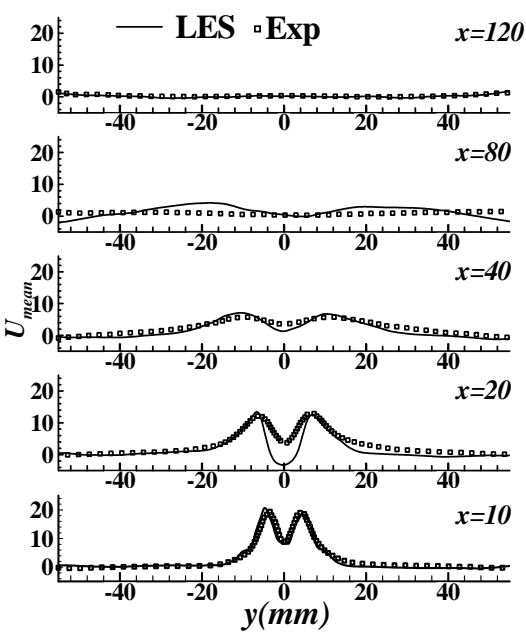

(b)

Fig. 4. Radial profiles of averaged axial velocities at different axial locations from the exit of swirler (unit mm). (a) for the case 1. (b) for the case 2.

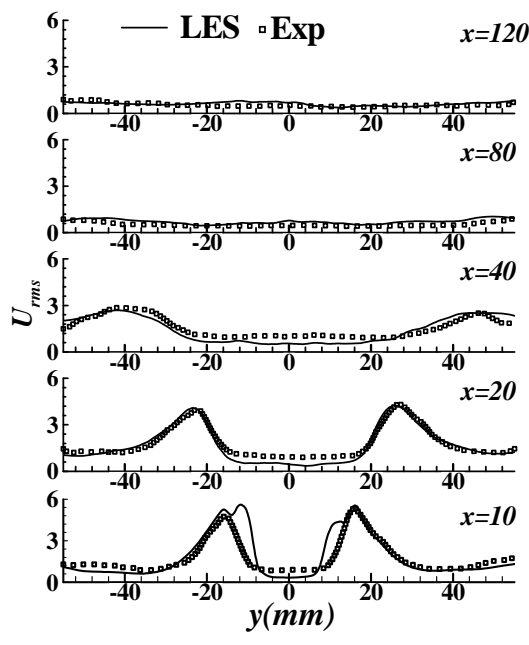

(a)

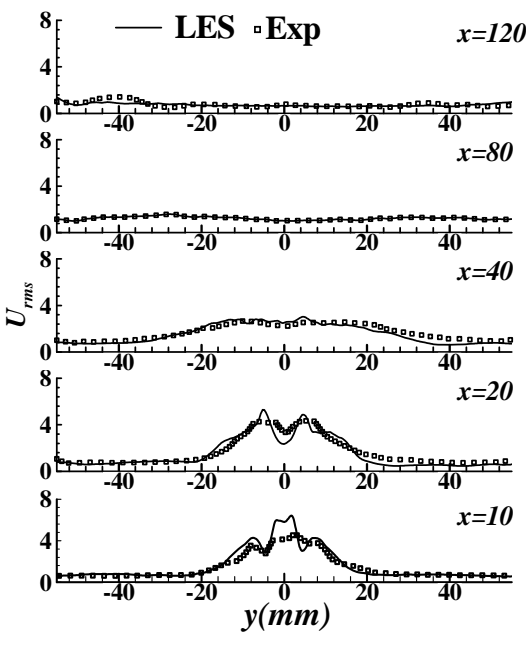

(b)

Fig. 5. Radial profiles of rms fluctuation of axial velocity at different axial locations from the exit of swirler (unit mm). (a) for the case 1. (b) for the case 2.

By calculating and analyzing the rms velocity, the turbulent characteristics of the flow field in the combustion chamber can be analyzed, and its distribution can also reflect the instability of the flow field in the combustion chamber. It can be seen from Fig. 5 (a) that in the case 1 , at $x=10 \mathrm{~mm}$, two large peaks appear in the axial rms velocity. This location is close to the exit of the swirler so that large swirl strength and relatively large velocity fluctuation in the flow field are observed. Comparison of the LES profiles with experimental profiles shows that LES results display two more peaks, although they are relatively small. The discrepancy could be resulted from the not fine enough grid resolution across the strong shear region. By comparing Fig. 4 (b) and Fig. 5 (b), it can be seen that where the velocity is higher, the rms velocity gradually increases, and the distribution of the rms velocity profile is not uniform, which also indicates that the unsteady flow field in the combustion chamber is more obvious where the swirl intensity is relatively strong. The peak value represents the position of the shear layer of the airflow in the combustion chamber. In the downstream locations, the radial distance between 


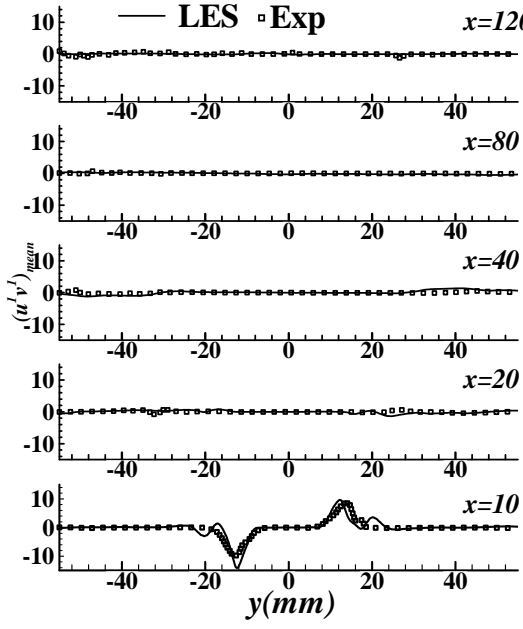

(a)

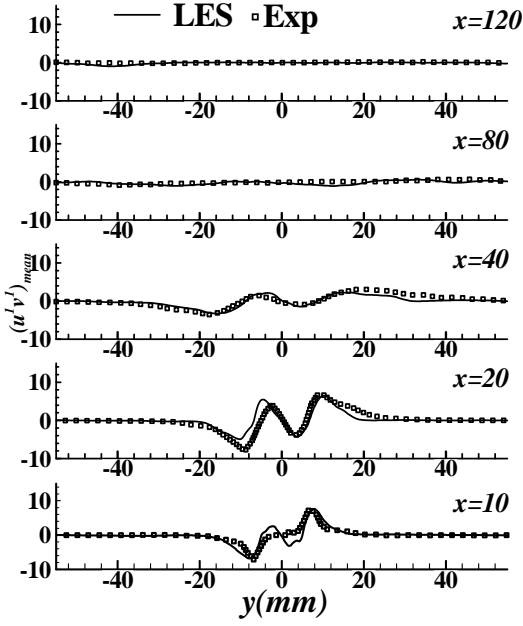

(b)

Fig. 6. Radial distribution of shear stress at different axial locations from the exit of swirler (unit mm). (a) for the case 1 . (b) for the case 2 .

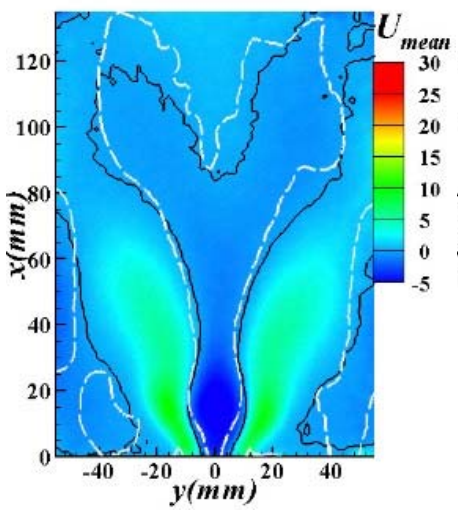

(a)

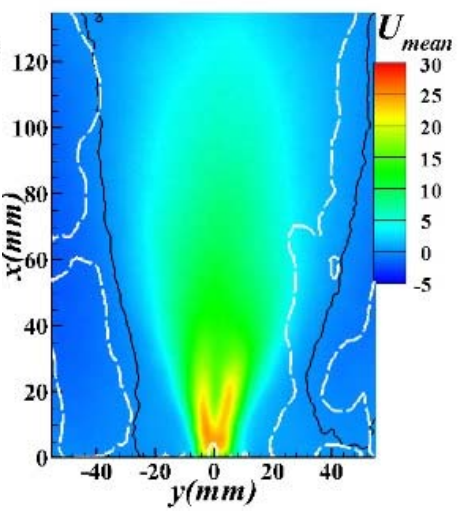

(b)

Fig. 7. Contour of averaged velocity. (a) for the case 3. (b) for the case 4.

the two peaks gradually increases, which represents that the position of the shear layer spread outwards with the airflow under the influence of swirl, and the rms velocity decreases with the decrease in turbulent fluctuations.

The shear stress also provide characterization of the turbulent fluctuations in the flow field. Figure 6 displays the distribution for shear stress along the radial directions at different axial locations for the case 1 and case 2. At $x=10 \mathrm{~mm}$, in the Fig. 6 (a), shear stresses are attributed by large swirl intensity and velocity fluctuations. Along the downstream directions, the shear stress becomes much smaller and more evenly distributed, which indicates that the flow field in the combustion chamber gradually develops completely. Note that at $x=10 \mathrm{~mm}$, peak in case 2 is smaller than that at $x=20 \mathrm{~mm}$. And along the downstream directions, the shear stress gradually decreases, which indicates that under the affection of vortex, Shear stress is extended at further downstream locations.

\subsection{Influence of volume flow rate}

For the constant flow rate conditions, the cases 3 and 4 are considered. Ratio of $R$ value between these two cases is more than 5. In this way, it is important to study both the flow field characteristics and the flow field variation of the two cases caused by the velocity ratio. Figure 7 (a) shows the contour of averaged velocity field in the case 3 , where the black and white solid lines in the axial velocity field, represent the iso-surface of zero velocity obtained from experiments and LES, respectively. It is observed that the flow area is expanded on both sides, leading to 
the center recirculation zone as "gourd" shape. The angular recirculation zone reaches the middle of the combustion chamber for both sides. The area of angular recirculation zone is large, and the recirculation zone is roughly symmetrical along the central axis. Certainly, such asymmetric recirculation zone is also required by the stable flow field.

Figure 7 (b) shows the instantaneous contour of averaged velocity field in the case 4 . It can be seen that there is no recirculation zone in the center of the combustor in the case 4 . The velocity ratio of the inner to outer tube is different than the case 3 , which gives a different radial velocity distribution for the case 4 .

Figure 8 shows the comparison between the LES and experimental data through the radial profiles of averaged axial velocities at different axial locations from the exit of swirler $(x=10,20,40,80$ and $120 \mathrm{~mm}$ ) for the case 3 and the case 4 . The excellent consistency between experimental and LES results is observed, even better than the case 1 and case 2 (Fig. 4). Comparison between the LES and experimental results through the radial profiles of rms axial velocity at different axial locations for the case 3 and the case 4 is shown in Fig. 9. It can be inferred that the radial profile of rms axial velocity in the case 3 is roughly the same as that in the case 1 (seen in Fig. 5). At the exit of the swirler, presence of the velocity gradients between the inner swirl and the outer swirl form two shear layers: one is between the inner swirl and the central recirculation zone, and the other is between the outer swirl and the inner swirl. Along the downstream directions, the peak values of axial velocity gradually disappear for both the cases, representing the distribution of the fully developed flow.

Shear stress distributions in the radial direction of the experiment and LES results for the case 3 and the case 4 are shown in Fig. 10. It can be seen from Fig.10 (a) that the Shear stress displays two peaks in the case 3 .
The first peak appears for the shear layer between inner swirl and the central reflux regions, while the outer peak displays a shear layer between the inner and outer swirl regions. As discussed before, there are two shear layers in the combustion chamber, which are accurately predicted here. At $x=10 \mathrm{~mm}$, LES values are over predicted than the experimental results, but qualitatively calculated results are in good agreement. The comparison of Fig. 7 (b) and Fig.10 (b) shows that no clear reflux appears under relatively large volume of inner swirl. And also, the radial distribution of Shear stress is low in the middle but high on both the sides. It indicates that although large velocity fluctuation exists, but in the case of weak swirl intensity, no coupling phenomenon appears for axial velocity and circumferential velocity, and it is not able to produce strong enough negative pressure zone.

\subsection{Effect of " $R$ "}

" $R$ " represents the balance relation between the relative magnitude of internal and external swirling momentum, whose information helps us to learn more about the development of flow field. There have been many previous studies of coaxial jets with different velocity ratios (Warda et al. 1999, Ribeiro and Whitelaw 1980, Erina et al. 2002), but there are few studies about the characteristic of flow field in a swirling burner with different velocity ratios. In this work, the velocity ratio of inner and outer tubes increases in order of the case 1, 3,2 and 4, leading to a considerable increase of rotational momentum of inner pipe and shrink of center reflux. This phenomenon has been analyzed to some extent in Fig. 3 and 7. With increase in " $R$ " values, the rotational momentum increases for outer tube, while the corresponding rotational momentum of the inner tube decreases. This leads to a continuous shrinkage of the central recirculation zone until it begins to dissipate, as shown in Fig. 3 (b) of the case 2. As the case 4 has the maximum velocity ratio of inner and outer tubes, its flow field characteristics should be

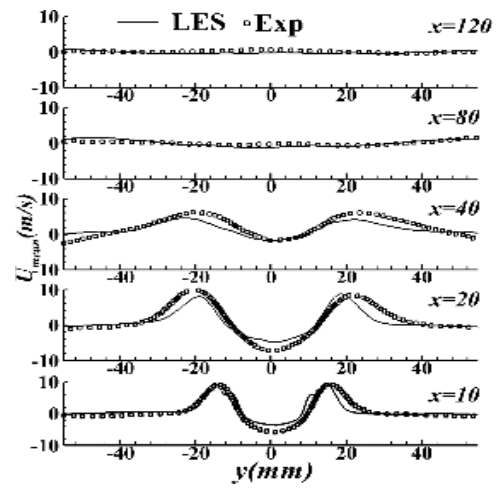

(a)

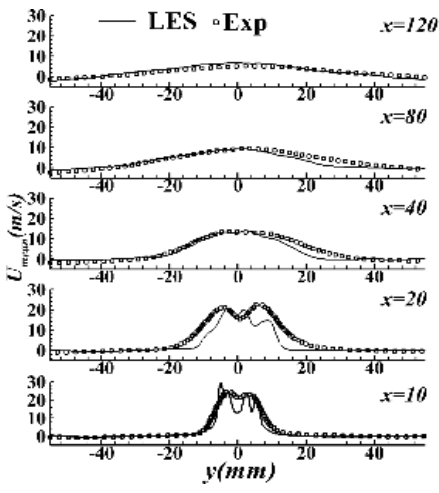

(b)

Fig. 8. Radial profiles of averaged axial velocities at different axial locations from the exit of swirler (unit mm). (a) for the case 3. (b) for the case 4. 


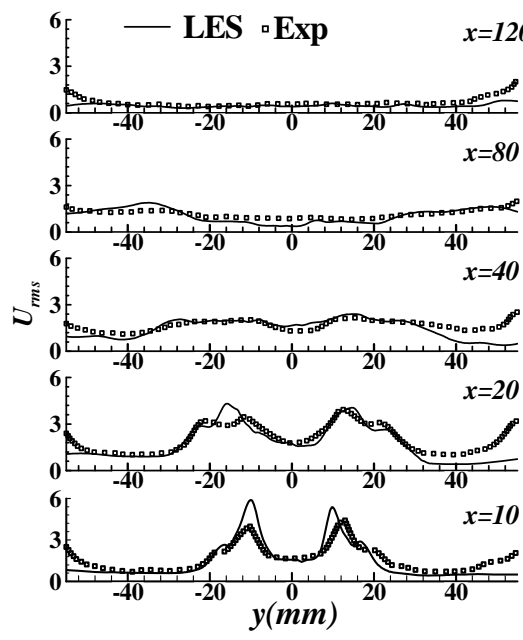

(a)

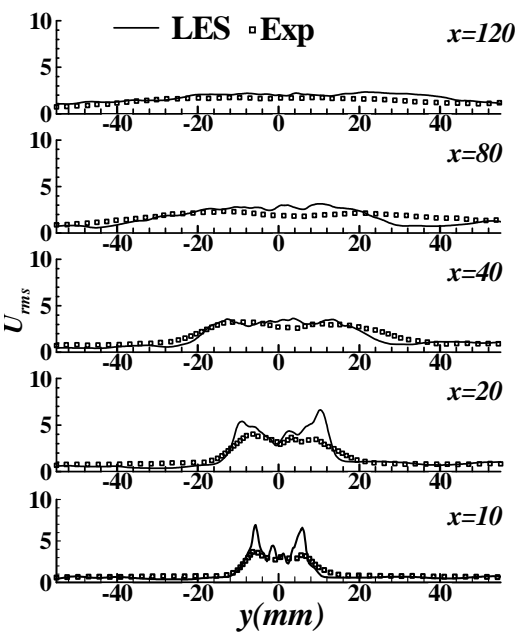

(b)

Fig. 9. Radial profiles of rms fluctuation of axial velocity at different axial locations from the exit of swirler (unit mm). (a) for the case 3. (b) for the case 4.

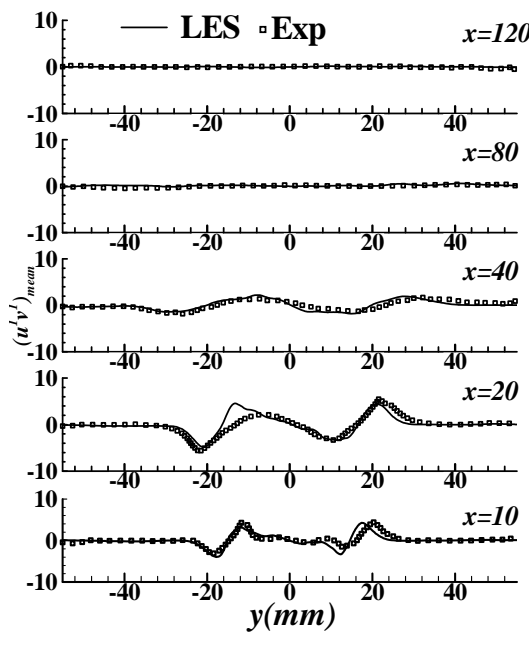

(a)

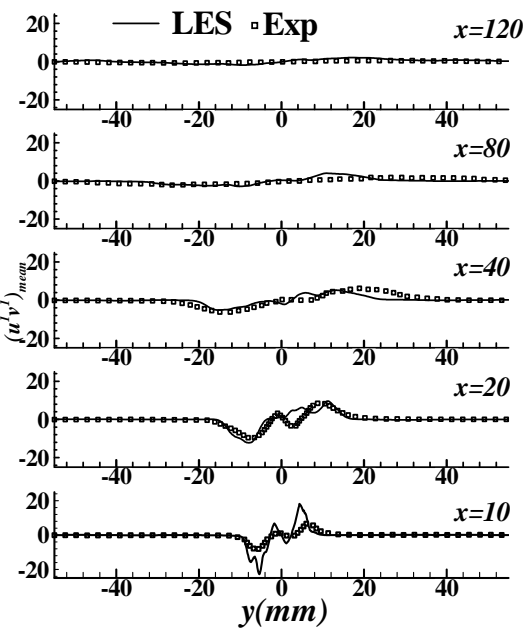

(b)

Fig. 10. Radial distribution of shear stress at different axial locations from the exit of swirler (unit mm). (a) for the case 3. (b) for the case 4.

completely different from that of the case 1 and the case 3 when the outer tube has a large rotational momentum. Moreover, the momentum difference between inner and outer tubes has increased in the case 2 so the central recirculation zone may not even occur. This phenomenon has been confirmed in Fig. 7.

Such a trend can be seen more clearly in Fig. 11, which shows the mean streamlines in the center plane in the order of increase in $R$ values. As the velocity of the outer tube is higher than that of the inner tube, the high-speed rotary jet is issued from the external swirler, which has a relatively larger rotational momentum, forming an obvious low-pressure central recirculation zone. And it can also be seen clearly that the vortex center distance and the length of the central reflux area in the angular backflow area. In the case 3 , value of $R$ is greater than the case 1 but, in this case, the inner and outer tube velocities are equal, resulting in the rotational equivalent ratio of the gas issued from inner and outer tubes is closer than that of the case 1 . Compared to the case 1 , in the case 3 , the recirculation zone has a larger length and a reduced density, although reflux also occurs on the both sides of the combustion chamber, i.e. the angular recirculation zone described above. In the 


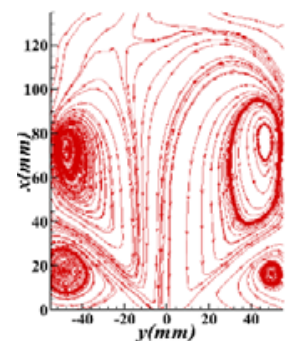

(a)

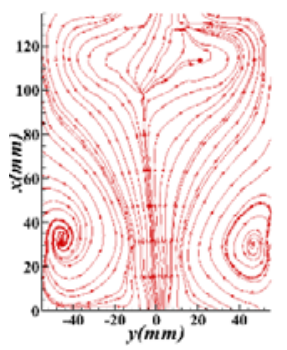

(c)

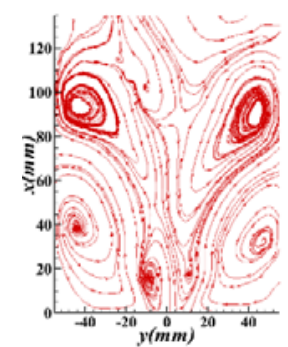

(b)

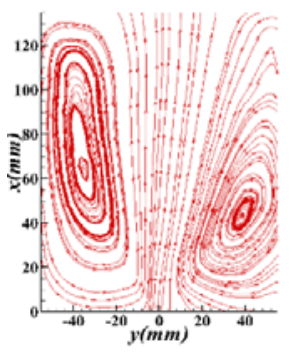

(d)

Fig. 11. Mean streamlines in the center plane for the four cases: (a) case 1 , (b) case 2 , (c) case 3 , (d) case 4 .

downstream, the reflux phenomenon of the airflow presents relatively obvious distribution on both sides of the central axis. However, as the airflow at the center cannot form a strong negative pressure region under the condition of weak swirling, the reflux region is not present compared to the case 1 . In addition, near swirler exit, local reflux is generated under the action of low pressures, forming a selfrecirculation zone except for the airflow flowing back from the downstream. It happens, because the internal and external rotational momentums are closer in the case 3 than the case 1 . It is noted that the flow field of the case 2 continues to shrink along the downstream directions until it almost disappears. Only some small negative velocity regions appear near the exit. Certainly, on both sides of the wall, there is still a local negative velocity area also called as angular reflux area which continuously expands. Finally, when $R$ value increases to 5 in the case 4, the velocity of the inner tube is much higher than that of the outer tube. It can be clearly seen that the recirculation zone completely disappears in the center and downstream regions of the case 4 . On contrary, the angular recirculation zone increases. The high-speed airflow appears as backflow on both the sides because it is affected by the upper wall of the combustor. The air flow propagates along the wall until it reaches the bottom of the combustor, and then it mixes with the air flow issued from the swirler.

\subsection{Coherent structure analysis}

In order to check the vortex structure in the flow field, a commonly used method, namely $\mathrm{Q}$ criterion

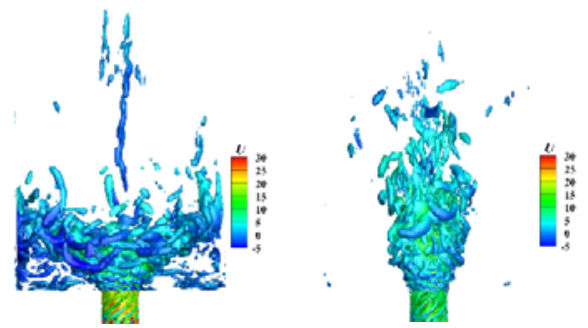

(a)

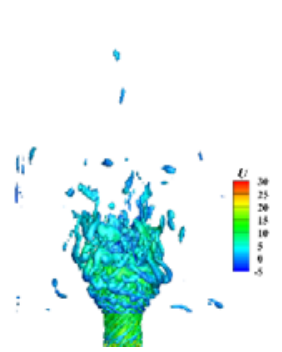

(c) (b)

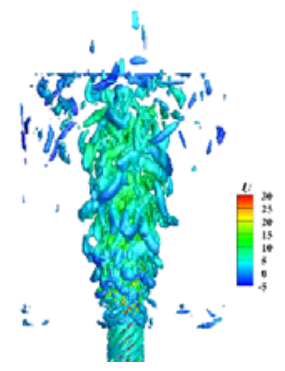

(d)
Fig. 12. $Q$ criterion iso-surface colored with axial velocity (U). (a) case 1 . (b) case 2 . (c) case 3 . (d) case 4.

(Kunnen et al. 2010, Zhao et al. 2014), is adopted in this paper. $\mathrm{Q}$ criterion identifies vortices of a $3 \mathrm{D}$ incompressible flow as connected fluid regions represents the second-order invariant of velocity gradient (Jeong and Hussain 1995. Kolá and ístek 2015). In the research of a swirl burner, it is usually focused on the macro vortex flow, and the value $Q$ should not be given too large. In this paper, value $\mathrm{Q}$ is chosen as about $0.02 \%$ of the maximum value found inside the flow field, for obtaining a better view of the coherent structures for all the four cases. Figure 12 shows the iso-surface of $\mathrm{Q}$ criterion for all the four cases, with the value $\mathrm{Q}=500,000$, which is colored with axial velocity $U$. All the four cases show different vortex structures, where the Q criterion isosurface represents a good vortex shape. This is a very effective method in identifying the vortexes. The vortex does not display an obvious spiral shape, but the large-scale vortices are seen in the flow field, which are originated from the exit of the swirler. These large-scale vortices represent the shear layer between the outer tube swirl and the inner tube swirl. At the exit of swirler, the peripheral flow field starts to break down into small vortexes, which are randomly distributed around the large-scale vortexes. In the downstream locations, the large-scale vortex structure rapidly expands and breaks the randomly distributed small vortexes, resulting in the formation of the central reflux region that is also a kind of vortex breakdown. For the case 2 and case 4, the generated vorticity distribution is relatively concentrated, mainly near the middle and upper regions, corresponding to the flow velocity of the inner and outer tubes. However, in the case 3, which 
is a case of the strong swirling, the outer laminar flow field also starts to break into small vortex clusters near the swirler exit, that are randomly distributed in the whole flow field. The inner vortex takes a single spiral shape, and it originates from the exit of the swirler. However, at the downstream locations, the swirling effect weakens, and the large-scale vortexes break into small randomly distributed vortexes. At further downstream, these randomly distributed vortexes gradually disappear.

By comparing the vortex shapes of the all four conditions, it is found that there are different vortex structures, but none of them has obvious characteristics of PVC (Precession vortex core). When the velocity between the inner and outer tubes is the same, the vortices are mainly distributed in the upstream regions of the combustion chamber, and the flow field is relatively wide. For the large Reynolds number of inner tube, the vortex distribution reaches further downstream, and even reaches the exit of the combustion chamber.

In every case, the spiral vortex structure can be found, but it is not easy to identify whether there is the PVC or spiral vortex. Therefore, in order to accurately describe the PVC phenomenon, the instantaneous distributions of pressure, streamline and circumferential velocity are plotted in the Fig. 13 for all the four cases at $x=10 \mathrm{~mm}$ form the swirler exit. In the case 1, there is no PVC phenomenon. From the instantaneous distribution, it is found that most of the fluid is deviated from the central axis, and rotates around the vortex axis. The location of the vortex is marked with black circles in the instantaneous circumferential velocity distribution, where the circumferential velocity at the vortex axis is zero. It is seen in the instantaneous pressure distribution that the position of the vortex is not at the lowest pressure because the local low pressure area is often produced by the vortex structure, which means that the minimum pressure does not meet the characteristics of PVC. Therefore, we can say that the case 1 does not possess the phenomenon of PVC at $x=10 \mathrm{~mm}$. Similarly, the analyses for remaining three cases do not indicate a presence of the PVC phenomenon.

\section{Conclusion}

In this paper, we perform experiments and LES on a newly designed two-stage axial swirler. LES calculations and PIV measurements are carried out and compared for the four different cold state conditions. In addition, the coherent structure of the simulated three-dimensional flow field is also analyzed. The main conclusions drawn from the current study are as follows.

LES results are found to be in a good agreement with experimental results. Small discrepancy between LES and experimental results is thought to be due to the not fine enough grid resolution across the strong shear layer. In the case 1, a large central reflux region appears, while in the case 2, only a small and scattered recirculating zone appears away from the exit of the swirler. It is analyzed to be due to the difference in gas density and velocity which results in a small distribution of circumferential velocity on the outside and a large distribution in the inside for the case 2. The overall rotational momentum also decreases in the case 2, which resists the formation of the low-pressure region. In addition, under the condition of constant volume flow rate, the velocity of the outflow from the inner tube is equal to that of outer tube in the case 3 , while the velocity of the outflow from inner tube is much higher than that of the outer tube in the case 4 . A large central reflux area appears in the case 3 . On contrary, the circumferential velocity cannot be coupled with the axial velocity and no central reflux area and partial reflux areas on both sides of the combustion chambers are shown in the case 4 .

The analysis of rms velocity and shear stress radial profiles show that two shear layers present, one is between the flow corresponds to inner and outer tubes in the upstream regions of the flow field, and other between the central recirculation zone and the main flow. Moreover, at the exit of the swirler, the shear stress is relatively large, which enhances the mixing phenomenon.
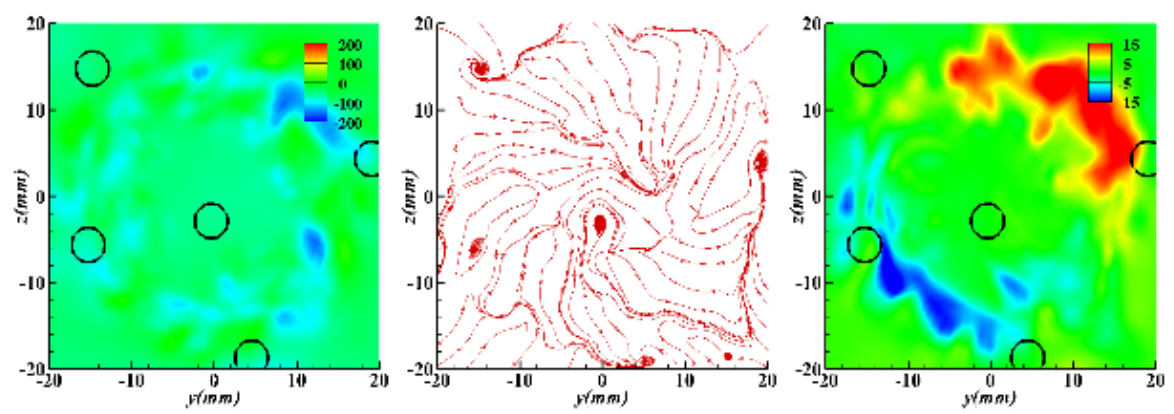

Fig. 13. Instantaneous distribution of pressure, streamline and circumferential velocity from left to right to analyze the presence of PVC phenomenon in the flow field of the case 1. 
With the increase in $\mathrm{R}$ values, the flow issued from outer tube gets smaller rotation momentum. So that the central reflux area gradually shrinks and finally disappears from the combustion chamber, while the length and scope of the angular reflux area continuously increase until it reaches the bottom of the combustion chamber. Furthermore, the analyses of the coherent structures for all the four cases show that under the conditions of central recirculation zone, vortex breakdown mainly occurs near the exit of the swirler, and with this, the vortex structure develops along the axial and radial direction. Under the condition of higher outflow velocity from the inner tube, the vortex can extend farther downstream, and it can even reach the exit of combustion chamber. At last, the LES analyses do not indicate the presence of PVC phenomenon in all four considered cases.

\section{Acknowledgement}

The authors thank the support of Natural Science Foundation of China (NSFC) through the projects: 91741117 and 51576092, and the support of Hightech Research Key laboratory of Zhenjiang (SS2018002). The computation work is conducted in the HPC Center of Jiangsu University.

\section{References}

Adawy, M. E., M. R. Heikal, A. Aziz, S. Munir and M. I. Siddiqui (2018). Effect of boost pressure on the in-cylinder tumble-motion of gdi engine under steady-state conditions using stereoscopic-piv. Journal of Applied Fluid Mechanics 11(3), 733-742.

Archer, S. and A. Gupta (2004). The Role of Confinement on Flow Dynamics Under Fuel Lean Combustion Conditions, International Energy Conversion Engineering Conference.

Arthur, K. (2018). Porous media flow transitioning into the forchheimer regime: a piv study. Journal of Applied Fluid Mechanics 11(2), 297-307.

Beer, J. M. and N. A. Chigier (1972). Combustion Aerodynamics. London: Applied Science Publishers Ltd.

Cai, J., S. M. Jeng and R. Tacina (2005) The Structure of a Swirl-Stabilized Reacting Spray Issued from an Axial Swirler, 43rd AIAA Aerospace Sciences Meeting and Exhibit.

Canepa, E., P. D. Martino, P. Formosa, M. Ubaldi and P. Zunino (2006). Unsteady aerodynamics of an aeroengine double swirler lean premixing prevaporizing burner. Journal of Engineering for Gas Turbines \& Power 128(1), 29-39.

Eldrainy, Y. A., K. M. Saqr, H. S. Aly, T. M. Lazim and M. N. M. Jaafar (2011). Large eddy simulation and preliminary modeling of the flow downstream a variable geometry swirler for gas turbine combustors. International Communications in Heat \& Mass Transfer 38(8), 1104-1109.

El-Kady, A., S. M. Jeng and H. Mongia (2005). Experimental Investigation of Temperature and Species Concentrations Characteristics of Swirling Spray Combustion, 43rd AIAA Aerospace Sciences Meeting and Exhibit.

El-Kady, A., S. M. Jeng and H. Mongia (2006). The Influence of Primary Air Jets on Flow and Pollutant Emissions Characteristics within a Model Gas Turbine Combustor, 44th AIAA Aerospace Sciences Meeting and Exhibit.

Murakami, E. and D. Papamoschou. (2002). Mean flow development in dual-stream compressible jets. AIAA Journal 40(6), 1131-1138.

Hadef, R. and B. Lenze (2008). Effects of co- and counter-swirl on the droplet characteristics in a spray flame. Chemical Engineering \& Processing Process Intensification 47(12), 2209-2217.

Houben, J., C. Weiss, E. Brunnmair and S. Pirker (2016). Cfd simulations of pressure drop and velocity field in a cyclone separator with central vortex stabilization rod. Journal of Applied Fluid Mechanics.

Huang, Y. and V. Yang (2005). Effect of swirl on combustion dynamics in a lean-premixed swirl-stabilized combustor. Proceedings of the combustion institute 30(2), 1775-1782.

Jeong, J. and F. Hussain (1995). On the identification of a vortex. Journal of Fluid Mechanics 332(1), 339-363.

Kim, H. S., V. K. Arghode and A. K. Gupta (2009). Flame characteristics of hydrogen-enriched methane-air premixed swirling flames. International Journal of Hydrogen Energy 34(2), 1063-1073.

Kim, J. C., K. H. Yoo and H. G. Sung (2011). Largeeddy simulation and acoustic analysis of a turbulent flow field in a swirl-stabilized combustor. Journal of Mechanical Science and Technology 25(10), 2703-2710.

Kolá, V. and J. ístek (2015). Corotational and compressibility aspects leading to a modification of the vortex-identification qcriterion. Aiaa Journal 53(8), 1-5.

Kunnen, R. P. J., H. J. H. Clercx, and B. J. Geurts (2010). Vortex statistics in turbulent rotating convection. Physical Review E 82(3), 1-12.

Lu, X., S. Wang, H. G. Sung, S. Y. Hsieh and V. Yang (2005). Large-eddy simulations of turbulent swirling flows injected into a dump chamber. Journal of Fluid Mechanics 527, 171-195.

Manikandan, R., R. Sadanandan and C. Prathap 
P. Wang et al. / JAFM, Vol. 15, No. 2, pp. 325-336, 2022

(2020). Experimental investigation on the effects of swirl on the exit turbulent flow field of an unconfined annular burner at isothermal and reacting conditions. Journal of Applied Fluid Mechanics 13(3), 839-847.

Raj, R. T. K. and V. Ganesan (2008). Study on the effect of various parameters on flow development behind vane swirlers. International Journal of Thermal Sciences 47(9), 1204-1225.

Reddy, A. P., R. I. Sujith and S. R. Chakravarthy (2006). Swirler Flow Field Characteristics in a Sudden Expansion Combustor Geometry. Journal of Propulsion \& Power 22(4), 800-808.

Ribeiro, M. M. and J. H. Whitelaw (1980).Coaxial jets with and without swirl. Journal of Fluid Mechanics.

Stopper, U., M. Aigner, W. Meier, R. Sadanandan and I. S. Kim (2009). Flow Field and Combustion Characterization of Premixed Gas Turbine Flames by Planar Laser Techniques. Journal of Engineering for Gas Turbines and Power 131(2), 32-39.

Tiwari, P., Z. Xia and X. Han (2020). Comparison of vles and les turbulence modeling for swirling turbulent flow. Journal of Applied Fluid Mechanics 13(4), 1107-1116.

Vashahi, F., S. Lee and J. Lee (2017a). Experimental and Computational Analysis of the Swirling Flow Generated by an Axial Counter-Rotating Swirler in a Rectangular Model Chamber
Using Water Test Rig, Journal of Engineering for Gas Turbines and Power: Transactions of the ASME 139 (8).

Vashahi, F., B. J. Baek and J. Lee (2017b). An experimental and LES comparison of waterand air-based swirling flow test rigs in vertical and horizontal configurations, Journal of Mechanical Science and Technology 31 (7), 3285-3295.

Warda, H. A., S. Z. Kassab, K. A. Elshorbagy and E. A. Elsaadawy (1999). An experimental investigation of the near-field region of free turbulent round central and annular jets. Flow Measurement \& Instrumentation 10(1), 1-14.

Westerweel, J., G. E. Elsinga, and R. J. Adrian (2013). Particle image velocimetry for complex and turbulent flows. Annual Review of Fluid Mechanics 45(1), 409.

Wu, Y., C. Carlsson, R. Szasz, L. Peng, L. Fuchs, X. S. Bai (2016). Effect of geometrical contraction on vortex breakdown of swirling turbulent flow in a model combustor. Fuel 170, 210-225.

Yang, Y. (2012). Large-eddy simulations of the nonreactive flow in the Sydney swirl burner. International Journal of Heat and Fluid Flow $36,47-57$.

Zhao, J. L., H. J. Wang, R. Z. Gong, T. Xuan and X. Y. Li (2014). Vortex structure research method based on Q-criterion in a high-speed centrifugal pump. 6th International Symposium on Fluid Machinery and Fluid Engineering. 